\title{
How Data Comm Methods and Multi-dimensional Traffic Displays Influence Pilot Workload under Trajectory Based Operations
}

\author{
Jimmy H. Nguyen ${ }^{1}$, L. Paige Bacon ${ }^{1}$, R. Conrad Rorie ${ }^{1}$, Meghann Herron ${ }^{1}$, \\ Kim-Phuong L. Vu ${ }^{1}$, Thomas Z. Strybel ${ }^{1}$, and Vernol Battiste ${ }^{2}$ \\ ${ }^{1}$ California State University Long Beach 1250 Bellflower Blvd., Long Beach, CA, USA \\ ${ }^{2}$ NASA Ames Research Center, Flight Deck Display Research Lab (FDDRL), \\ Moffet Field, CA, USA \\ \{Jimmy.Nguyen, Lauren.Bacon, Robert.Rorie\} astudent.csulb.com; \\ MeghannBrowne@gmail.com; \{kvu8,tstrybel\}@csulb.edu; \\ Vernol.Battiste-1@nasa.gov
}

\begin{abstract}
The goal of the present study was to examine the impact of different data-communication (Data Comm) methods and use of multi-dimensional displays (2-D or 3-D) on pilot workload when Trajectory Based Operations (TBO) are employed. Eight pilots flew simulated enroute flights using an integrated (FANS-1A) or non-integrated (ACARS) Data Comm method. Pilots were also asked to rate the workload and acceptability of a route modification with the different Data Comm methods. Online assessments during the flight simulation showed no difference in pilot ratings of workload and route acceptability. However, in post trial questionnaires, pilots reported an overall preference for FANS as a Data Comm method compared to ACARS. The display type did not change pilots' positive ratings for the FANS method, but 3D displays increase the operator's ability to understand the proposed flight plan changes when they used ACARS.
\end{abstract}

Keywords: Data Comm, 2-D displays, 3-D display, ACARS, FANS-1A, Trajectory Based Operations, Workload, NASA CSD.

\section{Introduction}

According to the FAA, by 2030, large airport hubs will see a $70.6 \%$ increase in passenger enplanements [1]. With this increase in demand, the capacity of the current radar-based, ground-centered air traffic management (ATM) system will be exceeded. Moreover, other limitations of the current ATM system such as the number of gates, runways, and airways will lead to delays, fuel inefficiency, and unsafe flying conditions. The Next Generation Air Transportation System (NextGen) is being developed to overcome these problems. NextGen encompasses a systematic overhaul of the air traffic control system based on new procedures and advanced technologies. These new technologies need to be evaluated to determine how they affect flight crew performance. The present study will evaluate two technologies, that of Data Comm and multi-dimensional displays. 
Currently, ATC-pilot communications are almost exclusively voice based, which may be inadequate for ensuring safe separation in the high-density traffic environments envisioned in the future. One solution for reducing radio communication congestion is to use pilot-controller Data Comm. Data Comm is a digital, text-based messaging system for sending trajectory changes between pilots and ATCs. In its simplest form, pilots will receive a text message and input the changes into their flight management system upon evaluation of the content for flight safety. However, if the Data Comm is integrated into the flight management system (FMS), these flight plan changes can be uploaded directly without the need for manual input.

Flight deck technology traditionally uses a 2-D top-down navigation display (NAV), accompanied by a primary flight display to indicate an aircraft's horizontal and vertical position. In NextGen, multi-view, 3-D displays can be integrated into the flight deck to allow pilots to visually inspect trajectory changes and make decisions based on them. Initial studies comparing 2-D versus 3-D displays on flight decks have shown that 2-D displays provided a more accurate representation of spatial information, and this allowed pilots to better make distance and spatial judgments. This performance benefit for 2-D displays was attributed to the fact that the display screens were 2-D. With 3-D displays, the axes must be compressed into a 2-D format which creates spatial ambiguity [3] [4] [5] [6]. However, more recent traffic displays, such as NASA's Flight Deck Display Research Laboratory's (FDDRL) Cockpit Situational Display (CSD) [7], provide the operator with a 3-D volumetric display capable of being manipulated. It has been shown to overcome spatial ambiguity issues associated with traditional 3-D displays. Moreover, there is evidence that this type of 3-D display can yield performance equivalent to that obtained with 2-D NAV displays [8].

One study that examined use of Data Comm with 2-D and 3-D displays showed that pilots had better judgment of their aircraft heading and position when flight path changes provided by the controller were integrated into a 3-D display [2]. The present study extends this work by examining the impact of different Data Comm methods and a multi-view display on pilot ratings of workload and route acceptability changes. We examined two Data Comm methods: (a) ACARS, a commercially available text messaging system that is present in most current-day commercial aircraft; and (b) FANS-1A (FANS), a more advanced, integrated Data Comm method, where route clearances can be uploaded directly into the FMS. With ACARS, the flight information is manually entered by pilots into the FMS. Because ACARS involves more steps for making flight path changes than FANS, it should require more time to complete a task, and may increase pilot workload. We also evaluated whether the type of graphical display, 2-D or 3-D, could affect operator workload and route acceptability ratings with each Data Comm method. Given that visual integration of flight path information increases operator's awareness of his/her position [2], it was hypothesized that, although ACARS may require more workload to implement a flight change than FANS, this workload could be mitigated with the integration of the flight path capability provided by a 3-D display. 


\section{Methods}

This study was part of a larger study called Separation Assurance using FANS-1 Automation for Resolution Implementation (SAFARI). The goal of SAFARI was to examine flight crew and ATC negotiations using Trajectory-Based Operations TBO procedures in the enroute phase of flight. SAFARI was conducted over a two week period. The present study reports data from the second week of the simulation, using eight specific trials where the participant flight crews flew simulated aircraft that were FANS-1A or ACARS equipped, with traditional 2-D displays. In addition, 8 trials were added to the end of the SAFARI simulation that enabled flight crews to use a 3D display, the NASA FDDRL CSD display.

\subsection{Participants}

Eight air transport pilots (6 Captains and 2 First Officers) participated as part of four flight crews. Five pilots reported having more than 3000 hours in "glass" cockpits while the remaining three reported having less than 3000 hours of glass cockpit experience. For Data Comm exposure, six of the eight pilots reported having experience communicating with non-integrated Data Comm (ACARS). Only two pilots reported having experience communicating with integrated Data Comm (FANS-1A) through actual flight time. These eight participants will be referred to as the "participant pilots" or "experimental flight decks". Other aircraft in the sector were flown by "pseudopilots", who were researchers working in the lab. There were also two radar-certified controllers that managed the active sectors, but the present study only reports data from the participant pilots.

\subsection{Design}

A 2 (Display type) x 2 (Data Comm type) factorial design was employed to examine the relationship between type of display and type of Data Comm method on pilot ratings of workload and route acceptability. For display type, pilots were presented with a fixed 2-D display similar to the current- day NAV display, with a PFD, on the first half of the trials, and the NASA FDDRL's 3-D CSD on the second half of the trials. For Data Comm type, integrated and non-integrated Data Comm systems were simulated. The integrated Data Comm system was modeled after FANS-1A; this system allowed route modifications to be loaded directly into the flight management system. The non-integrated Data Comm system, modeled after ACARS, required pilots to manually input route modifications into FMS.

\subsection{Dependent Variables}

Workload probes were presented in real time. Flight crews were queried individually on their current workload ( $1=$ low and $5=$ high) every two minutes; however, these workload probes were replaced by route acceptability probes based on the actions of the flight crew. If the flight crew executed a route modification, a route acceptability probe would be presented instead of a workload probe at the next interval. The route acceptability probe asked the flight crews to rate the acceptability of the recently 
executed route by judging the quality of the route modification based on a scale of 1 to $5(1=$ poor, $3=$ good, $5=$ best $)$. In addition to workload probes, post trial workload ratings were obtained from the participant pilots at the end of each simulation run.

\subsection{Apparatus}

The simulation environment was set up using the Multiple Aircraft Control System (MACS) developed by the NASA Ames Airspace Operations Laboratory [9], and the 3-D CSD developed by NASA Ames' FDDRL [7]. Four desktop pilot stations were used to simulate multiple low fidelity flight decks. Each station was manned by a flight crew of two pilots, one pilot serving the role of Captain and the other First Officer. Each station contained five displays:

- two standard monitors

- CSD (2-D or 3-D)

- PFD

- two individual touch screen displays

- Mode Control Panel (MCP)

- Probe panel to display workload and route acceptability probes

- one shared touch screen monitor with a MACS display.

These displays were used to mimic a flight deck environment. Each pilot shared a center touch screen display with a simulated Boeing $777 \mathrm{MCP}$, two CDUs, and an EICAS display used to alert pilots to incoming Data Comm messages. The shared touch screen display was used to emulate similar physical actions on an actual MCP and CDU interface. The touch screen monitor for the shared display was an ACER T230H model, with a 23" wide-screen TFT LCD. Additionally, each pilot had a monitor displaying a CSD and a PFD next to the shared touch screen display.

\subsection{FANS-1A and ACARS Procedures}

To integrate TBO with both methods of Data Comm, procedures on how to proceed under specific levels of flight deck equipage were developed for pilots and ATCs. For aircraft equipped with FANS-1A Data Comm, the flight crew responded to ATC uplinks of route modifications by accepting, loading, and executing the new flight plan, or rejecting it. Once accepted, the flight crew was able to examine the route modification via the CSD display (in either 2-D or 3-D format, depending on the condition) and could load this route into the FMS if it was appropriate, or reject it based on flight safety considerations. If the new route was acceptable, the flight crew could execute the new route. If this route was rejected, crews could request another route or propose one to the ATC via the FANS-1A procedures provided to them.

For aircraft equipped with ACARS, the procedure required the flight crew to manually input waypoints and/or lat-long coordinates that were uplinked by ATC into the appropriate leg of their flight plan through the use of the CDU. Once inputted, the crew examined the proposed route, and either accepted or rejected it based on flight safety. Since ACARS route modifications could not be directly loaded into the FMS, route modifications required the crew to key in new waypoints and lat-long 
coordinates into the CDU within a certain amount of time to make sure the aircraft stayed on course. If the flight crew could not approve and execute the proposed route modification in this time window, then a contingency flight plan was provided to them.

\subsection{Procedure}

Before the SAFARI experimental trials began, participant pilots and ATCs were briefed on their task, types of aircraft, Data Comm equipage, and TBO procedures. Then each pilot was assigned to a flight crew, as Captain or First Officer, based on their reported flight experience. In addition, each pilot was assigned the role of "pilot flying" or "pilot managing". The pilot managing role required the pilot to input ATC uplinks into the CDU. The pilot flying made the final decision on whether to accept or reject a proposed route. Both pilot roles were counter-balanced so each pilot was the pilot flying or pilot managing on an equal number of trials. After the roles were assigned, the flight crews were trained on the simulation configuration. The training modules addressed the flight deck set up, the functions of the MCP, CDU, CSD, PFD, radio control, pilot responsibilities, communications, and answering probe questions. Each module was followed by a skills test that required each pilot to answer questions about the module on which they were trained. Training modules were not considered completed until pilots successfully answered all skills test questions.

Once the training was completed, the experimental trials began. At the beginning of each trial, flight crews were assigned a communication equipage level for their aircraft, as well as given a reference guide for the specific Data Comm procedures to be used displays that required them to downlink a route modification request with ATC for weather avoidance. Flight crews were to follow their assigned Data Comm procedures when communicating with ATC, unless maintaining flight safety was at risk. In this case, they were to revert back to voice communication. With the FANS-1A Data Comm equipage, the crews were able to insert waypoints into the CDU to create conflict-free flight paths that were downlinked to the ATC. The ATC could then accept the proposed route, reject it, or reject it and propose a new flight plan. If the ATC accepted the route, the flight crew received an accepted message, prompting them to execute the flight plan via the CDU. If the ATC rejected the route, flight crews were able to propose another route or wait for a flight plan to be uplinked to them from the ATC. Under ACARS equipage, the flight crews constructed a flight plan by sending a free text message of to the ATC. The ATC would construct that route modification using a trial planner tool and then accept it or proposed another route to uplink back to the flight deck. If the ATC uplinked the requested route, the flight crew would follow ACARS procedure to accept the route and confirm it with ATC.

During each scenario, pilots and controllers were probed on their workload every two minutes. Availability of workload queries was signaled by an audio chime to alert the pilots of an awaiting workload question. Pilots responded to the workload queries by providing a rating of 1 to 5, with 1 being low workload and 5 being high workload. The probe station also presented event-triggered route acceptability queries instead of the workload queries. If the flight crews executed a route modification by pressing the "execute" button on the CDU, the next probe query would be a route acceptability probe instead of a workload probe. For the route 
acceptability ratings, the scale was also 1 to $5(1=$ poor, $3=$ Good, and $5=$ Best route modification). After each trial, pilots were also required to complete post trial questionnaires. Experimental observers were always on standby to assist flight crews with any procedural questions.

At the end of the SAFARI simulations, using the CSD in 2-D display mode, pilots were briefed on the use of the 3-D features of the CSD. Pilots were told that their flight responsibilities and the Data Comm procedures remained the same as in the SAFARI study. However, instead of viewing the weather, traffic, and flight plans using the 2-D view and PFD, pilots would be able to manipulate the 3-D CSD display with their computer mouse to rotate the airspace in any direction. In addition, the weather was displayed in a 3-D format as well, simulating NexRad weather. Pilots were trained on the 3-D CSD display before the experimental trials began. Workload and route acceptability probes, and the post trial questionnaires, were administered in the same manner as in the SAFARI study. Once pilots completed the supplemental trials, they completed a post simulation questionnaire.

\section{Results}

Workload ratings, route acceptability ratings and comments on post experimental trial questionnaires were analyzed. Separate 2 (Display) x 2 (Data Comm) ANOVAs were conducted on the pilots' ratings of workload and route acceptability during the experimental trials. The same ANOVA was performed on other ratings provided by the participant pilots on the post trial questionnaire relating to flight safety, efficiency, ability to communicate with ATC, and overall/peak workload for the enroute and for weather avoidance phases of flight.

\subsection{Workload and Route Acceptability}

Pilots' ratings of workload during the experimental trials did not yield a significant main effect for Data Comm method or display type, $F \mathrm{~s}<1.0$. In addition, no interaction of the two variables was obtained, $F<1.0$. Similarly, no significant effects were obtained for route acceptability ratings, $F_{\mathrm{s}}<1.0$. Table 1 displays mean ratings for workload and route acceptability for each Data Comm method and display type. Note that both the workload and acceptability ratings were low.

Table 1. Mean workload and acceptability ratings presented by Data Comm and display type. The standard error of the mean is in parenthesis.

\begin{tabular}{ccccc}
\hline \multicolumn{5}{c}{ Data Comm Type } \\
\multicolumn{5}{c}{ ACARS } \\
\hline 2-D & 3-D & FANS \\
Workload & $1.97(.17)$ & $1.98(.10)$ & $1.90(.12)$ & $1.93(.10)$ \\
Acceptability & $1.78(.24)$ & $1.85(.17)$ & $1.83(.17)$ & $1.67(.22)$ \\
\hline
\end{tabular}




\subsection{Post Trial Questionnaire}

Pilots were asked to rate their ability to communicate with ATC using the different Data Comm methods on a 1-5 scale, where 1 was low and 5 was high. In terms of effectiveness, there was an effect of Data Comm type, $F(1,7)=18.36, p<.01$. Pilots rated FANS $(M=4.23)$ significantly higher in effectiveness than ACARS $(M=3.81)$. For efficiency of communication, a marginally significant main effect for Data Comm was also present, $F(1,7)=5.11, p=.058$, where pilots reported that ATC communications were more efficient when using FANS $(M=4.23)$ than ACARS $(M=4.00)$.

For understandability, another marginal effect of Data Comm was obtained, $F(1,7)$ $=4.65, p=.068$. Pilot rated their understanding of ATC-issued flight plan clearances to be slightly better with FANS $(M=4.30)$ than ACARS $(M=4.00)$. However, this main effect was qualified by a significant interaction with display type, $F(1,7)=9.32$, $p<.05$, see Figure 1. Display type did not influence pilot understandability ratings of ATC communication with FANS. However, with ACARS, the 3D CSD display increased the pilot's ability to understand ATC trajectory changes compared to the 2D display. Taken together, these findings suggest that pilots using the FANS Data Comm had better understanding of ATC communication. However, using the 3-D multi-view display reduced the negative effects associated with ACARS relating to pilots' ability to understand ATC proposed flight path modifications.

Pilots also reported that overall workload differed between the Data Comm methods, $F(1,7)=8.41, p<.05$, with less workload reported for FANS $(M=1.98)$ than ACARS $(M=2.31)$. For overall workload in avoiding weather, a main effect for Data Comm type was also present, $F(1,7)=7.33, p<.05$. When using FANS, pilots again reported experiencing less workload $(M=1.97)$ than when using ACARS ( $M=$ 2.20).

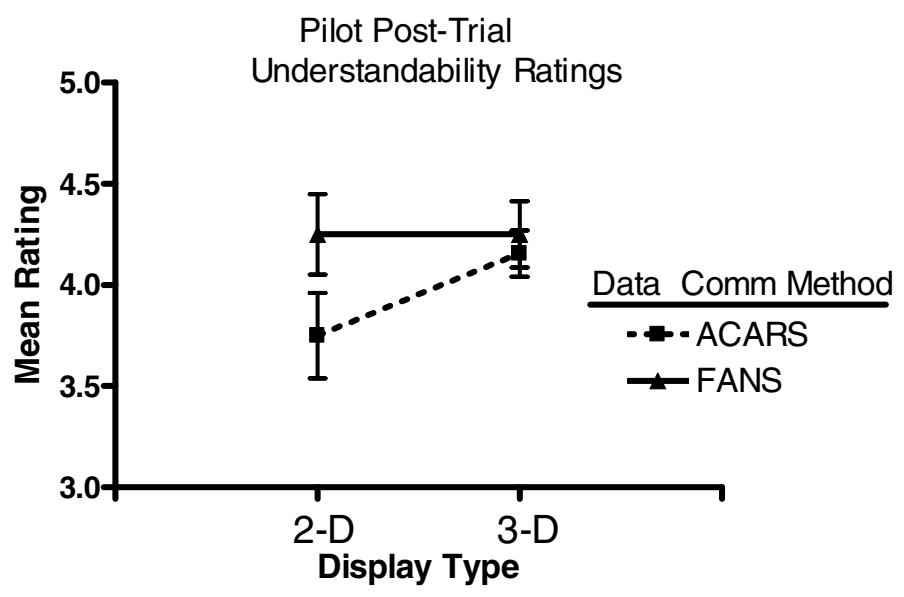

Fig. 1. Display by Data Comm interaction for rating $(1=$ low; $5=$ high $)$ of understandability of ATC clearances 


\section{Discussion}

Pilots reported higher workload levels associated with using the ACARS Data Comm method than with the FANS method when queried at the end of the trial, but not during the simulated flight. One possible explanation for the discrepant findings from the online probes versus post-trial results is that, with ACARS, pilots reverted back to voice communications when its use did not lead to timely actions during the simulation. As such, the pilots kept their workload at low, manageable levels throughout the scenario. The post-trial workload ratings likely reflected the pilots' preference for the FANS Data Comm method over ACARS. However, because operator preference for an interface does not necessarily lead to better performance with the interface [10], performance data with the different Data Comm methods need to be examined to determine the overall effectiveness of the different Data Comm methods.

Overall, pilots rated FANS higher than ACARS when queried about the effectiveness, efficiency of communication, and understandability of ATC clearances. These findings suggest that pilots would more likely adopt Data Comm if implemented with FANS than with ACARS. The benefit of using ACARS for Data Comm delivery, though, is that it is already available on most commercial aircraft and can serve as a starting point for Data Comm usage. Although ACARS was rated lower in terms of message understandability than FANS, this difference disappeared when it was used in conjunction with the 3-D CSD display. The greater understandability ratings observed with 3-D displays was attributed to the display allowing pilots to visualize and inspect the flight plan change. This initial finding indicates that 3-D CSDs are promising tools for NextGen environments, and future investigations should continue to examine the use of 3-D displays in aiding pilot decision making.

Acknowledgments. This study was supported in part by NASA cooperative agreement NNX09AU66A, Group 5 University Research Center: Center for the Human Factors in Advanced Aeronautics Technologies (Brenda Collins, Technical Monitor).

\section{References}

1. Terminal Area Forecast (TAF) Summary Report Fiscal Year 2009 - 2030 (PDF File), http: / / www. faa.gov

2. Wickens, C.D., Miller, S., Mingpo, T.: The implications of data-link for representing pilot request information on 2D and 3D air traffic control displays. In: 38th Hum. Fac. Erg. Soc. P., Nashville, TN, pp. 61-65 (1994)

3. Alexander, A.L., Wickens, C.D.: 3D navigation and integrated hazard display in advanced avionics: Performance, situation awareness, and workload. (Technical Report AHFD-0510/NASA-05-2), NASA Langley Research Center, Hampton (2005)

4. Boyer, B.S., Wickens, C.D.: 3D weather displays for aircraft cockpits. (Technical Report ARL-94-11/NASA-94-4), University of Illinois, Aviation Research Laboratory, Savoy (1994) 
5. Boeckman, K.J., Wickens, C.D.: The resolution and performance effects of threedimensional display rotation on local guidance and spatial awareness. (Technical Report ARL-01-4/NASA-01-3), University of Illinois, Aviation Research Laboratory, Savoy (2001)

6. Alexander, A.L., Wickens, C.D., Merwin, D.H.: Perspective and coplanar cockpit displays of traffic information: Implications for maneuver choice, flight safety, and workload. Int. J. Aviat. Psychol. 15(1), 1-21 (2005)

7. Granada, S., Dao, Q., Wong, D., Johnson, W.W., Battiste, V.: Development and integration of a human-centered volumetric cockpit situation display for distributed airground operations. In: Proceedings of the 13th International Symposium on Aviation Psychology, Oklahoma City, OK, pp. 229-284 (2005)

8. Thomas, L.C., Wickens, C.D.: Display dimensionality, conflict geometry, and time pressure effects on conflict detection and resolution performance using cockpit displays of traffic information. Int. J. Aviat. Psychol. 16(3), 315-336 (2006)

9. Prevot, T.: Exploring the many perspectives of distributed air traffic management: The Multi Aircraft Control System: MACS. In: International Conference on Human-Computer Interaction in Aeronautics, pp. 23-25. MIT, Cambridge (2002)

10. Vu, K.-P.L., Proctor, R.W.: Naïve and experienced judgments of stimulus-response compatibility: Implications for interface design. Ergonomics 46, 169-187 (2003) 